\title{
ACTIVE CONTROLler Design fOR Global CHAOS SYNCHRONIZATION OF HYPERCHAOTIC BAO AND HYPERCHAOTIC XU SYSTEMS
}

\author{
Sundarapandian Vaidyanathan ${ }^{1}$ and Suresh Rasappan ${ }^{2}$ \\ ${ }^{1}$ Research and Development Centre, Vel Tech Dr. RR \& Dr. SR Technical University \\ Avadi, Chennai-600 062, Tamil Nadu, INDIA \\ sundarvtulgmail.com \\ ${ }^{2}$ Department of Mathematics, Vel Tech Dr. RR \& Dr. SR Technical University \\ Avadi, Chennai-600 062, Tamil Nadu, INDIA \\ mrpsuresh83@gmail.com
}

\begin{abstract}
This paper investigates the global chaos synchronization of identical hyperchaotic Bao systems (Bao and Liu, 2008), identical hyperchaotic Xu systems (Xu, Cai and Zheng, 2009) and non-identical hyperchaotic Bao and hyperchaotic Xu systems. Active nonlinear control is the method adopted for the global chaos synchronization of the hyperchaotic systems addressed in this paper and the synchronization results have been established using Lyapunov stability theory. Since the Lyapunov exponents are not required for these calculations, the active nonlinear control method is very effective and convenient to achieve global chaos synchronization of identical and non-identical hyperchaotic Bao and hyperchaotic Xu systems. Numerical simulations have been provided to demonstrate the effectiveness of the synchronization results for the hyperchaotic Cao and hyperchaotic Xu systems.
\end{abstract}

\section{KEYWORDS}

Active Control, Nonlinear Control, Chaos Synchronization, Hyperchaos, Hyperchaotic Bao System, Hyperchaotic Xu System.

\section{INTRODUCTION}

Chaos is a very interesting and complex nonlinear phenomenon. The first chaotic attractor was discovered by Lorenz ([1], 1963), when he studied the atmospheric convection. Since then, chaos has been received extensive investigations and chaos phenomenon has been observed in a variety of fields including physical systems [2], chemical systems [3], ecological systems [4], secure communications [5-6], etc.

The seminal work on chaos synchronization problem was published by Pecora and Carroll ([7], 1990). From then on, chaos synchronization has been extensively and intensively studied in the last three decades [8-30].

In most of the chaos synchronization approaches, the master-slave or drive-response formalism is used. If a particular chaotic system is called the master or drive system and another chaotic system is called the slave or response system, then the idea of global chaos synchronization is to use the output of the master system to control the slave system so that the states of the slave system have the same amplitude but opposite signs as the states of the master system asymptotically. In other words, the differences of the states of the master and slave systems are designed to converge to zero asymptotically, when global chaos synchronization occurs. 
In the recent years, various schemes have been deployed for chaos synchronization such as PC method [2], OGY method [8], active control [9-12], adaptive control [13-15], backstepping design [16], sampled-data feedback [17], sliding mode control [18-20], etc. Recently, active control method has been applied to achieve anti-synchronization of two identical chaotic systems [21-22].

Hyperchaotic system is usually defined as a chaotic system with at least two positive Lyapunov exponents, implying that its dynamics are expanded in several different directions simultaneously. Thus, the hyperchaotic systems have more complex dynamical behaviour which can be used to improve the security of a chaotic communication system. Hence, the theoretical design and circuit realization of various hyperchaotic signals have become important research topics [23-27].

In this paper, we use active control to derive new results for the global chaos synchronization of identical hyperchaotic Bao systems ([28], 2008), identical hyperchaotic Xu systems ([29], 2009) and non-identical hyperchaotic Bao and hyperchaotic Xu systems.

This paper is organized as follows. In Section 2, we describe the problem statement and our methodology using Lyapunov stability theory. In Section 3, we discuss the global chaos synchronization of identical hyperchaotic Bao systems (2008) using active nonlinear control. In Section 4, we discuss the global chaos synchronization of identical hyperchaotic Xu systems (2009) using active nonlinear control. In Section 5, we discuss the global chaos synchronization of hyperchaotic Bao and hyperchaotic Xu systems using active nonlinear control. In Section 6, we summarize the main results obtained in this paper.

\section{Problem Statement and Our Methodology}

As the master or drive system, we consider the chaotic system described by

$$
\dot{x}=A x+f(x),
$$

where $x \in R^{n}$ is the state vector, $A$ is the $n \times n$ matrix of system parameters and $f: R^{n} \rightarrow R^{n}$ is the nonlinear part of the system.

As the slave or response system, we consider the following chaotic system described by

$$
\dot{y}=B y+g(y)+u,
$$

where $y \in R^{n}$ is the state of the slave system, $B$ is the $n \times n$ matrix of system parameters, $g: R^{n} \rightarrow R^{n}$ is the nonlinear part of the system and $u$ is the active controller to be designed.

If $A=B$ and $f=g$, then $x$ and $y$ are the states of two identical chaotic systems. If $A \neq B$ or $f \neq g$, then $x$ and $y$ are the states of two different chaotic systems.

For the global chaos synchronization of the chaotic systems (1) and (2) using active control, we design a state feedback controller $u$, which synchronizes the states of the master system (1) and the slave system (2) for all initial conditions $x(0), y(0) \in R^{n}$. 
Thus, we define the synchronization error as

$$
e=y-x
$$

Hence, the error dynamics is obtained as

$$
\dot{e}=B y-A x+g(y)-f(x)+u
$$

Thus, the global chaos synchronization problem is essentially to find a feedback controller (active controller) $u$ so as to stabilize the error dynamics (4) for all initial conditions, i.e.

$$
\lim _{t \rightarrow \infty}\|e(t)\|=0, \text { for all } e(0) \in R^{n}
$$

We use the Lyapunov stability theory as our methodology. We take as a candidate Lyapunov function

$$
V(e)=e^{T} P e,
$$

where $P$ is a positive definite matrix. Note that $V: R^{n} \rightarrow R$ is a positive definite function by construction.

If we find a feedback controller $u$ so that

$$
\dot{V}(e)=-e^{T} Q e,
$$

where $Q$ is a positive definite matrix, then $\dot{V}: R^{n} \rightarrow R$ is a negative definite function.

Thus, by Lyapunov stability theory [25], the error dynamics (4) is globally exponentially stable.

\section{SyNCHRONIZATION OF IDENTICAL HYPERCHAOTIC BAO SYSTEMS}

In this section, we discuss the synchronization of identical hyperchaotic Bao systems ([28], 2008).

As the master system, we consider the hyperchaotic Bao system described by

$$
\begin{aligned}
& \dot{x}_{1}=a\left(x_{2}-x_{1}\right)+x_{4} \\
& \dot{x}_{2}=c x_{2}-x_{1} x_{3} \\
& \dot{x}_{3}=x_{1} x_{2}-b x_{3} \\
& \dot{x}_{4}=k x_{1}+d x_{2} x_{3}
\end{aligned}
$$

where $x_{1}, x_{2}, x_{3}, x_{4}$ are the state variables and $a, b, c, d, k$ are positive constants. 
International Journal of Information Technology Convergence and Services (IJITCS) Vol.1, No.6, December 2011

As the slave system, we consider the controlled hyperchaotic Bao dynamics described by

$$
\begin{aligned}
& \dot{y}_{1}=a\left(y_{2}-y_{1}\right)+y_{4}+u_{1} \\
& \dot{y}_{2}=c y_{2}-y_{1} y_{3}+u_{2} \\
& \dot{y}_{3}=y_{1} y_{2}-b y_{3}+u_{3} \\
& \dot{y}_{4}=k y_{1}+d y_{2} y_{3}+u_{4}
\end{aligned}
$$

where $y_{1}, y_{2}, y_{3}, y_{4}$ are the state variables and $u_{1}, u_{2}, u_{3}, u_{4}$ are the active controls.

The four-dimensional Bao system (11) is hyperchaotic when

$$
a=36, \quad b=3, c=20, d=0.1 \text { and } k=21 \text {. }
$$

The hyperchaotic portrait of the Bao system is shown in Figure 1.
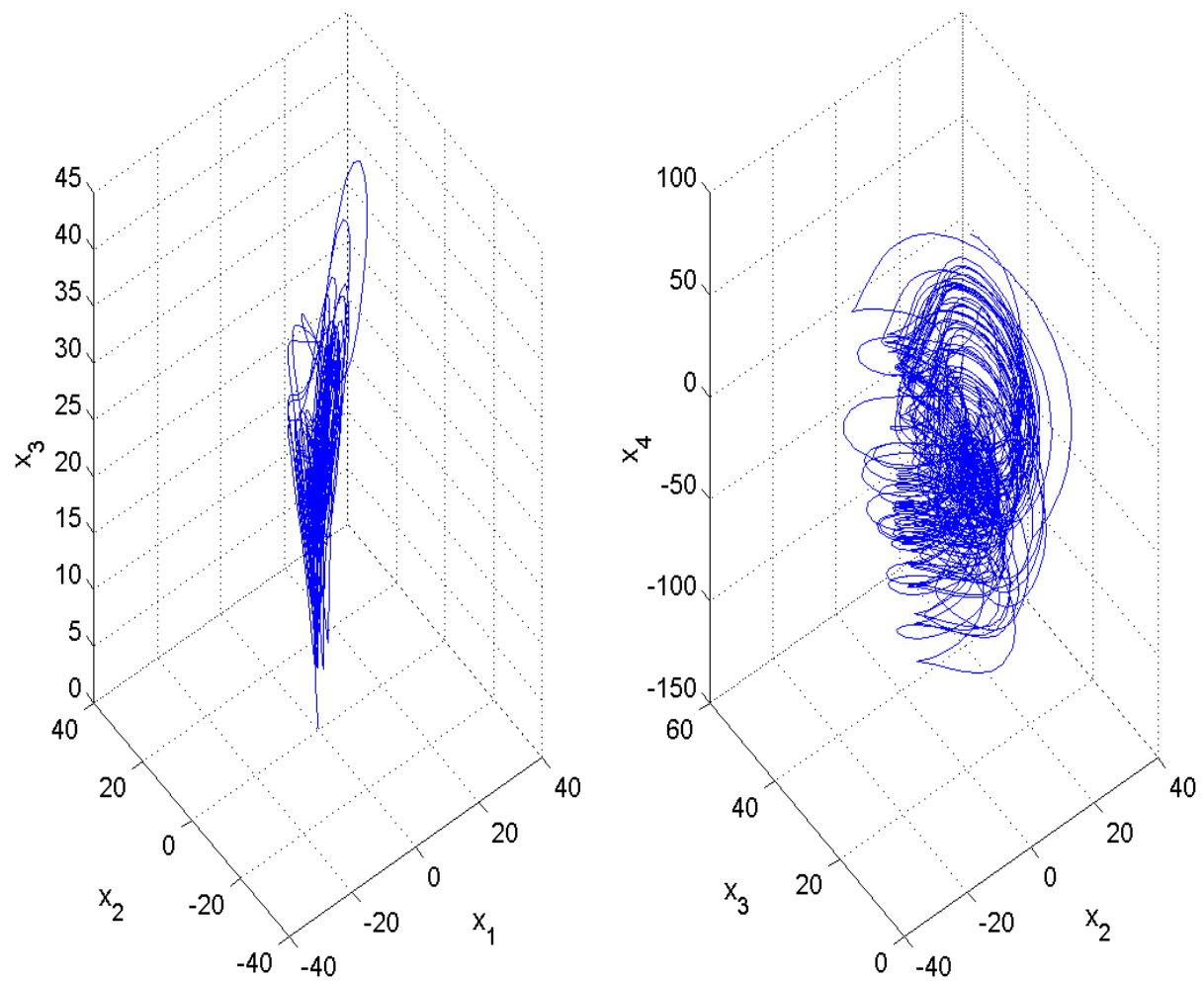

Figure 1. State Orbits of the Hyperchaotic Bao System

The synchronization error is defined as

$$
e_{i}=y_{i}-x_{i}, \quad(i=1,2,3,4)
$$


A simple calculation gives the error dynamics

$$
\begin{aligned}
& \dot{e}_{1}=a\left(e_{2}-e_{1}\right)+e_{4}+u_{1} \\
& \dot{e}_{2}=c e_{2}-y_{1} y_{3}+x_{1} x_{3}+u_{2} \\
& \dot{e}_{3}=-b e_{3}+y_{1} y_{2}-x_{1} x_{2}+u_{3} \\
& \dot{e}_{4}=k e_{1}+d\left(y_{2} y_{3}-x_{2} x_{3}\right)+u_{4}
\end{aligned}
$$

We consider the active nonlinear controller defined by

$$
\begin{aligned}
& u_{1}=-a e_{2}-e_{4} \\
& u_{2}=-(c+1) e_{2}+y_{1} y_{3}-x_{1} x_{3} \\
& u_{3}=-y_{1} y_{2}+x_{1} x_{2} \\
& u_{4}=-k e_{1}-e_{4}-d\left(y_{2} y_{3}-x_{2} x_{3}\right)
\end{aligned}
$$

Substitution of (12) into (11) yields the linear error dynamics

$$
\dot{e}_{1}=-a e_{1}, \quad \dot{e}_{2}=-e_{2}, \quad \dot{e}_{3}=-b e_{3}, \quad \dot{e}_{4}=-e_{4}
$$

We consider the quadratic Lyapunov function defined by

$$
V(e)=\frac{1}{2} e^{T} e=\frac{1}{2}\left(e_{1}^{2}+e_{2}^{2}+e_{3}^{2}+e_{4}^{2}\right)
$$

which is a positive definite function on $R^{4}$.

Differentiating (14) along the trajectories of the error system (13), we get

$$
\dot{V}(e)=-a e_{1}^{2}-e_{2}^{2}-b e_{3}^{2}-e_{4}^{2},
$$

which is a negative definite function on $R^{4}$ since $a$ and $b$ are positive constants.

Thus, by Lyapunov stability theory [25], the error dynamics (13) is globally exponentially stable. Hence, we obtain the following result.

Theorem 1. The identical hyperchaotic Bao systems (8) and (9) are globally and exponentially synchronized with the active nonlinear controller (12).

\section{Numerical Simulations}

For the numerical simulations, the fourth order Runge-Kutta method with initial timestep $h=10^{-6}$ is used to solve the two systems of differential equations (8) and (9) with the nonlinear controller (12).

The parameters of the identical hyperchaotic Bao systems (8) and (9) are selected as

$$
a=36, b=3, c=20, d=0.1 \text { and } k=21 .
$$


International Journal of Information Technology Convergence and Services (IJITCS) Vol.1, No.6, December 2011

The initial values for the master system (8) are taken as

$$
x_{1}(0)=5, \quad x_{2}(0)=8, x_{3}(0)=12, \quad x_{4}(0)=21
$$

and the initial values for the slave system (9) are taken as

$$
y_{1}(0)=14, \quad y_{2}(0)=20, \quad y_{3}(0)=18, \quad y_{4}(0)=12
$$

Figure 2 depicts the synchronization of the identical hyperchaotic Bao systems (8) and (9).


Figure 2. Synchronization of Identical Hyperchaotic Bao Systems

\section{SyNChronization OF IDENTICAL HyPERCHAOTIC Xu SYSTEMS}

In this section, we discuss the synchronization of identical hyperchaotic Xu systems ([29], 2009).

As the master system, we consider the hyperchaotic Xu system described by

$$
\begin{aligned}
& \dot{x}_{1}=\alpha\left(x_{2}-x_{1}\right)+x_{4} \\
& \dot{x}_{2}=\beta x_{1}+r x_{1} x_{3} \\
& \dot{x}_{3}=-\gamma x_{3}-l x_{1} x_{2} \\
& \dot{x}_{4}=x_{1} x_{3}-m x_{2}
\end{aligned}
$$

where $x_{1}, x_{2}, x_{3}, x_{4}$ are the state variables and $\alpha, \beta, \gamma, r, l, m$ are positive constants. 
International Journal of Information Technology Convergence and Services (IJITCS) Vol.1, No.6, December 2011

As the slave system, we consider the controlled hyperchaotic Xu dynamics described by

$$
\begin{aligned}
& y_{1}=\alpha\left(y_{2}-y_{1}\right)+y_{4}+u_{1} \\
& y_{2}=\beta y_{1}+r y_{1} y_{3}+u_{2} \\
& y_{3}=-\gamma y_{3}-l y_{1} y_{2}+u_{3} \\
& y_{4}=y_{1} y_{3}-m y_{2}+u_{4}
\end{aligned}
$$

where $y_{1}, y_{2}, y_{3}, y_{4}$ are the state variables and $u_{1}, u_{2}, u_{3}, u_{4}$ are the active controls.

The four-dimensional Xu system (16) is hyperchaotic when

$$
\alpha=10, \quad \beta=40, \quad \gamma=2.5, \quad r=16, \quad l=1 \text { and } m=2 \text {. }
$$

The hyperchaotic portrait of the $\mathrm{Xu}$ system is shown in Figure 3.
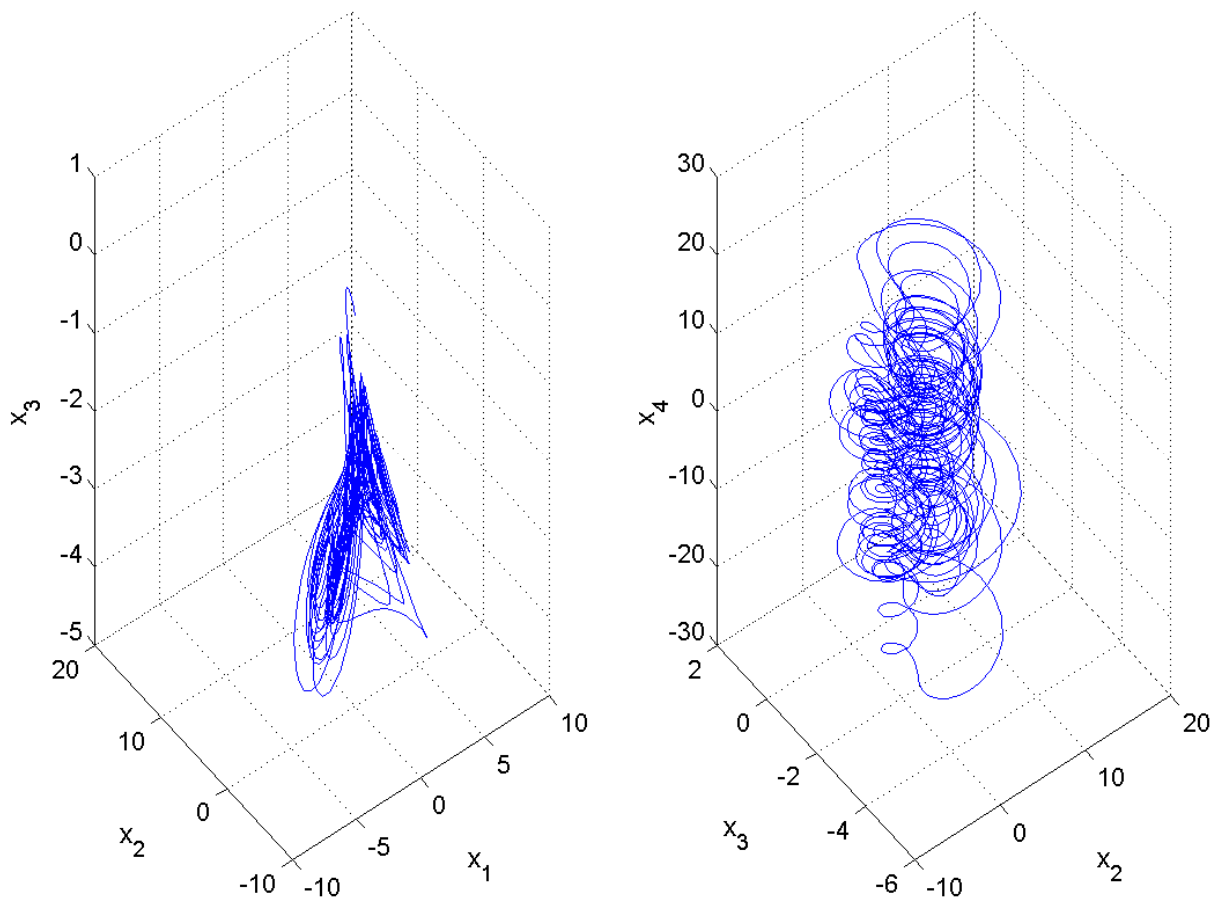

Figure 3. State Orbits of the Hyperchaotic Xu System

The synchronization error is defined as

$$
e_{i}=y_{i}-x_{i}, \quad(i=1,2,3,4)
$$


A simple calculation gives the error dynamics

$$
\begin{aligned}
& \dot{e}_{1}=\alpha\left(e_{2}-e_{1}\right)+e_{4}+u_{1} \\
& \dot{e}_{2}=\beta e_{1}+r\left(y_{1} y_{3}-x_{1} x_{3}\right)+u_{2} \\
& \dot{e}_{3}=-\gamma e_{3}-l\left(y_{1} y_{2}-x_{1} x_{2}\right)+u_{3} \\
& \dot{e}_{4}=-m e_{2}+y_{1} y_{3}-x_{1} x_{3}+u_{4}
\end{aligned}
$$

We consider the active nonlinear controller defined by

$$
\begin{aligned}
& u_{1}=-\alpha e_{2}-e_{4} \\
& u_{2}=-\beta e_{1}-e_{2}-r\left(y_{1} y_{3}-x_{1} x_{3}\right) \\
& u_{3}=l\left(y_{1} y_{2}-x_{1} x_{2}\right) \\
& u_{4}=m e_{2}-e_{4}-y_{1} y_{3}+x_{1} x_{3}
\end{aligned}
$$

Substitution of (20) into (19) yields the linear error dynamics

$$
\dot{e}_{1}=-\alpha e_{1}, \quad \dot{e}_{2}=-e_{2}, \quad \dot{e}_{3}=-\gamma e_{3}, \dot{e}_{4}=-e_{4}
$$

We consider the quadratic Lyapunov function defined by

$$
V(e)=\frac{1}{2} e^{T} e=\frac{1}{2}\left(e_{1}^{2}+e_{2}^{2}+e_{3}^{2}+e_{4}^{2}\right)
$$

which is a positive definite function on $R^{4}$.

Differentiating (22) along the trajectories of the error system (21), we get

$$
\dot{V}(e)=-\alpha e_{1}^{2}-e_{2}^{2}-\gamma e_{3}^{2}-e_{4}^{2},
$$

which is a negative definite function on $R^{4}$ since $\alpha$ and $\gamma$ are positive constants.

Thus, by Lyapunov stability theory [25], the error dynamics (21) is globally exponentially stable. Hence, we obtain the following result.

Theorem 2. The identical hyperchaotic Xu systems (16) and (17) are globally and exponentially synchronized with the active nonlinear controller (20).

\section{Numerical Simulations}

For the numerical simulations, the fourth order Runge-Kutta method with initial timestep $h=10^{-6}$ is used to solve the two systems of differential equations (16) and (17) with the nonlinear controller (20).

The parameters of the identical hyperchaotic Xu systems (16) and (17) are selected as

$$
\alpha=10, \quad \beta=40, \quad \gamma=2.5, \quad r=16, \quad l=1 \text { and } m=2 .
$$


International Journal of Information Technology Convergence and Services (IJITCS) Vol.1, No.6, December 2011

The initial values for the master system (16) are taken as

$$
x_{1}(0)=12, \quad x_{2}(0)=14, \quad x_{3}(0)=7, \quad x_{4}(0)=18
$$

and the initial values for the slave system (17) are taken as

$$
y_{1}(0)=26, \quad y_{2}(0)=10, \quad y_{3}(0)=18, \quad y_{4}(0)=24
$$

Figure 4 depicts the synchronization of the identical hyperchaotic Xu systems (16) and (17).
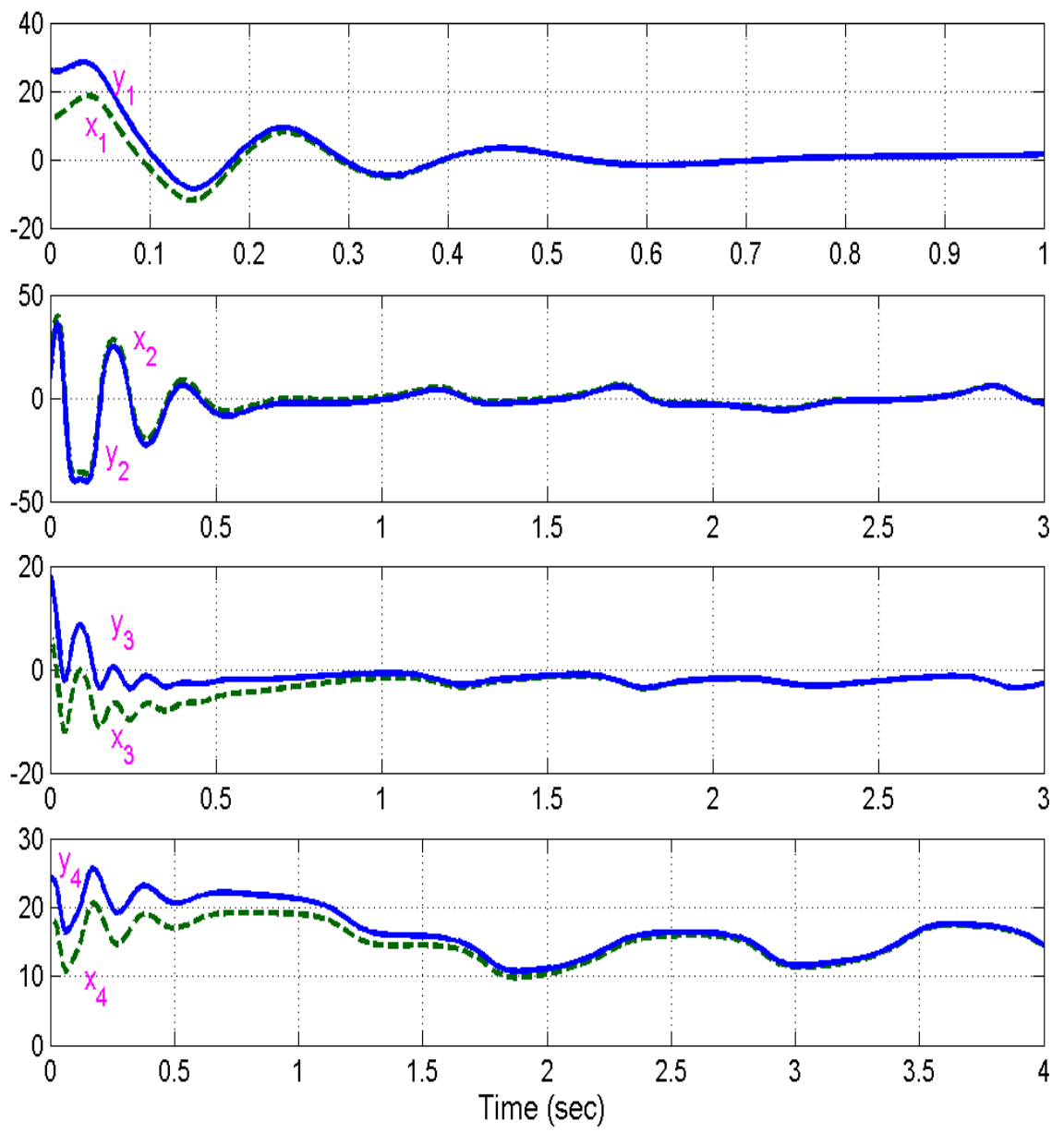

Figure 2. Synchronization of Identical Hyperchaotic Xu Systems 


\section{SYNCHRONizATION OF NON-IDENTICAL HyPERCHAOTIC BAO AND HYPERCHAOTIC XU SYSTEMS}

In this section, we consider the global chaos synchronization of non-identical hyperchaotic Bao system ([28], 2008) and hyperchaotic Xu system ([29], 2009).

As the master system, we consider the hyperchaotic Bao system described by

$$
\begin{aligned}
& \dot{x}_{1}=a\left(x_{2}-x_{1}\right)+x_{4} \\
& \dot{x}_{2}=c x_{2}-x_{1} x_{3} \\
& \dot{x}_{3}=x_{1} x_{2}-b x_{3} \\
& \dot{x}_{4}=k x_{1}+d x_{2} x_{3}
\end{aligned}
$$

where $x_{1}, x_{2}, x_{3}, x_{4}$ are the state variables and $a, b, c, d, k$ are positive constants.

As the slave system, we consider the controlled hyperchaotic Xu dynamics described by

$$
\begin{aligned}
& y_{1}=\alpha\left(y_{2}-y_{1}\right)+y_{4}+u_{1} \\
& y_{2}=\beta y_{1}+r y_{1} y_{3}+u_{2} \\
& y_{3}=-\gamma y_{3}-l y_{1} y_{2}+u_{3} \\
& y_{4}=y_{1} y_{3}-m y_{2}+u_{4}
\end{aligned}
$$

where $y_{1}, y_{2}, y_{3}, y_{4}$ are the state variables, $\alpha, \beta, \gamma, r, l, m$ are positive constants and $u_{1}, u_{2}, u_{3}, u_{4}$ are the active controls.

The synchronization error is defined as

$$
e_{i}=y_{i}-x_{i}, \quad(i=1,2,3,4)
$$

A simple calculation gives the error dynamics

$$
\begin{aligned}
& \dot{e}_{1}=\alpha\left(e_{2}-e_{1}\right)+e_{4}+(\alpha-a)\left(x_{2}-x_{1}\right)+u_{1} \\
& \dot{e}_{2}=\beta e_{1}+\beta x_{1}-c x_{2}+r y_{1} y_{3}+x_{1} x_{3}+u_{2} \\
& \dot{e}_{3}=-\gamma e_{3}+(b-\gamma) x_{3}-l y_{1} y_{2}-x_{1} x_{2}+u_{3} \\
& \dot{e}_{4}=-m e_{2}-k x_{1}-m x_{2}+y_{1} y_{3}-d x_{2} x_{3}+u_{4}
\end{aligned}
$$

We consider the active nonlinear controller defined by

$$
\begin{aligned}
& u_{1}=-\alpha e_{2}-e_{4}-(\alpha-a)\left(x_{2}-x_{1}\right) \\
& u_{2}=-\beta e_{1}-e_{2}-\beta x_{1}+c x_{2}-r y_{1} y_{3}-x_{1} x_{3} \\
& u_{3}=-(b-\gamma) x_{3}+l y_{1} y_{2}+x_{1} x_{2} \\
& u_{4}=m e_{2}-e_{4}+k x_{1}+m x_{2}-y_{1} y_{3}+d x_{2} x_{3}
\end{aligned}
$$


International Journal of Information Technology Convergence and Services (IJITCS) Vol.1, No.6, December 2011

Substitution of (28) into (27) yields the linear error dynamics

$$
\dot{e}_{1}=-\alpha e_{1}, \quad \dot{e}_{2}=-e_{2}, \quad \dot{e}_{3}=-\gamma e_{3}, \quad \dot{e}_{4}=-e_{4}
$$

We consider the quadratic Lyapunov function defined by

$$
V(e)=\frac{1}{2} e^{T} e=\frac{1}{2}\left(e_{1}^{2}+e_{2}^{2}+e_{3}^{2}+e_{4}^{2}\right)
$$

which is a positive definite function on $R^{4}$.

Differentiating (30) along the trajectories of the error system (29), we get

$$
\dot{V}(e)=-\alpha e_{1}^{2}-e_{2}^{2}-\gamma e_{3}^{2}-e_{4}^{2},
$$

which is a negative definite function on $R^{4}$ since $\alpha$ and $\gamma$ are positive constants.

Thus, by Lyapunov stability theory [25], the error dynamics (29) is globally exponentially stable. Hence, we obtain the following result.

Theorem 3. The non-identical hyperchaotic Bao system (24) and hyperchaotic Xu system (25) are globally and exponentially synchronized with the active nonlinear controller (28).

\section{Numerical Simulations}

For the numerical simulations, the fourth order Runge-Kutta method with initial timestep $h=10^{-6}$ is used to solve the two systems of differential equations (24) and (25) with the nonlinear controller (28).

The parameters of the identical hyperchaotic Bao system (24) are selected as

$$
a=36, b=3, c=20, d=0.1 \text { and } k=21 \text {. }
$$

The parameters of the identical hyperchaotic Xu system (25) are selected as

$$
\alpha=10, \quad \beta=40, \quad \gamma=2.5, \quad r=16, \quad l=1 \text { and } m=2 .
$$

The initial values for the master system (24) are taken as

$$
x_{1}(0)=25, \quad x_{2}(0)=6, \quad x_{3}(0)=9, \quad x_{4}(0)=14
$$

and the initial values for the slave system (25) are taken as

$$
y_{1}(0)=12, \quad y_{2}(0)=18, \quad y_{3}(0)=10, \quad y_{4}(0)=29
$$

Figure 5 depicts the synchronization of the non-identical hyperchaotic systems, viz. hyperchaotic Bao system (24) and hyperchaotic Xu system (25). 

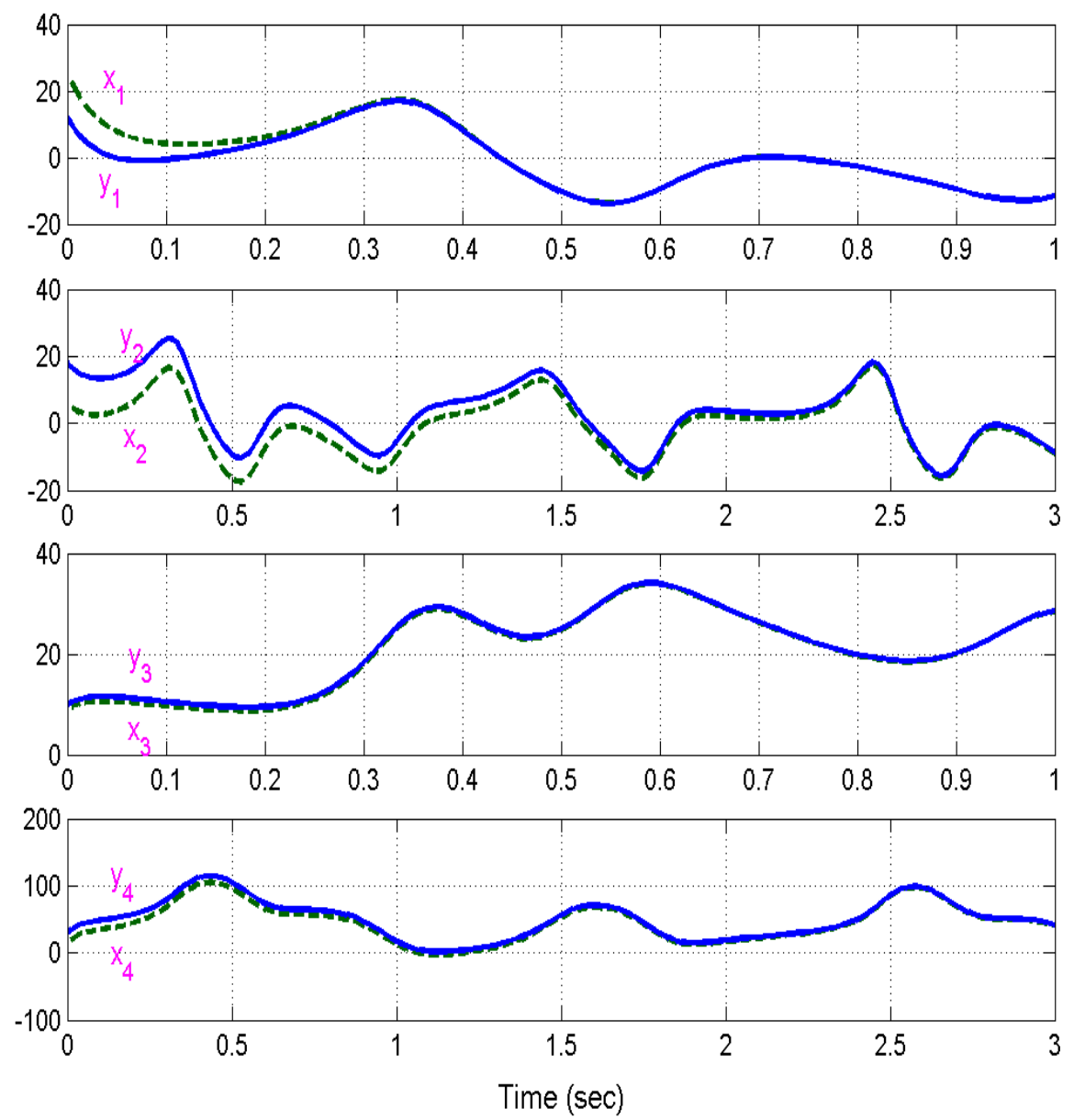

Figure 5. Synchronization of Hyperchaotic Bao and Hyperchaotic Xu Systems

\section{CONCLUSIONS}

It is well-known that the hyperchaotic systems are chaotic systems with at least two positive Lyapunov exponents and that the hyperchaotic systems have more complex dynamical behaviour than chaotic systems. Thus, the hyperchaotic systems can be used to improve the security of a chaotic communication system. In this paper, using the active control method, new results have been derived for the global chaos synchronization for the identical hyperchaotic Bao systems (2008), identical hyperchaotic Xu systems (2009) and non-identical hyperchaotic Bao and hyperchaotic Xu systems. The global synchronization results derived in this paper have been proved using Lyapunov stability theory. Since the Lyapunov exponents are not required for these calculations, the active control method is very convenient and efficient for the global chaos synchronization of identical and non-identical hyperchaotic Bao and hyperchaotic Xu systems. Numerical simulation results have been presented to illustrate the global chaos synchronization results derived in this paper. 
International Journal of Information Technology Convergence and Services (IJITCS) Vol.1, No.6, December 2011

\section{REFERENCES}

[1] Lorenz, E.N. (1963) “Deterministic nonperiodic flow”, J. Atmos. Sci., Vol. 20, pp 130-141.

[2] Lakshmanan, M. \& Murali, K. (1996) Nonlinear Oscillators: Controlling and Synchronization, World Scientific, Singapore.

[3] Han, S.K., Kerrer, C. \& Kuramoto, Y. (1995) "Dephasing and burstling in coupled neural oscillators", Phys. Rev. Lett., Vol. 75, pp 3190-3193.

[4] Blasius, B., Huppert, A. \& Stone, L. (1999) "Complex dynamics and phase synchronization in spatially extended ecological system”, Nature, Vol. 399, pp 354-359.

[5] Feki, M. (2003) "An adaptive chaos synchronization scheme applied to secure communication", Chaos, Solitons and Fractals, Vol. 18, pp 141-148.

[6] Murali, K. \& Lakshmanan, M. (1998) "Secure communication using a compound signal from generalized synchronizable chaotic systems”, Phys. Rev. Lett. A, Vol. 241, pp 303-310.

[7] Pecora, L.M. \& Carroll, T.L. (1990) "Synchronization in chaotic systems", Phys. Rev. Lett., Vol. 64, pp 821-824.

[8] Ott, E., Grebogi, C. \& Yorke, J.A. (1990) “Controlling chaos”, Phys. Rev. Lett., Vol. 64, pp 1196-1199.

[9] Ho, M.C. \& Hung, Y.C. (2002) "Synchronization of two different chaotic systems by using generalized active control", Physics Letters A, Vol. 301, pp. 424-428.

[10] Chen, H.K. (2005) "Global chaos synchronization of new chaotic systems via nonlinear control”, Chaos, Solitons \& Fractals, Vol. 23, pp. 1245-1251.

[11] Sundarapandian, V. (2011) "Global chaos synchronization of four-scroll and four-wing chaotic attractors by active nonlinear control," International Journal on Computer Science and Engineering, Vol. 3, No. 5, pp. 2145-2155.

[12] Sundarapandian, V. (2011) "Anti-synchronization of Arneodo and Coullet systems by active nonlinear control," International Journal of Control Theory and Applications, Vol. 4, No. 1, pp. 25-36.

[13] Liao, T.L. \& Tsai, S.H. (2000) "Adaptive synchronization of chaotic systems and its applications to secure communications", Chaos, Solitons and Fractals, Vol. 11, pp 1387-1396.

[14] Sundarapandian, V. (2011) "Adaptive control and synchronization of hyperchaotic Cai system", International Journal of Control Theory and Computer Modelling, Vol. 1, No. 1, pp. 1-13.

[15] Sundarapandian, V. (2011) "Adaptive synchronization of hyperchaotic Lorenz and hyperchaotic Liu systems", International Journal of Instrumentation and Control Systems, Vol. 1, No. 1, pp. $1-18$.

[16] Yu, Y.G. \& Zhang, S.C. (2006) “Adaptive backstepping synchronization of uncertain chaotic systems”, Chaos, Solitons and Fractals, Vol. 27, pp 1369-1375.

[17] Yang, T. \& Chua, L.O. (1999) "Control of chaos using sampled-data feedback control”, Internat. J. Bifurcat. Chaos, Vol. 9, pp 215-219.

[18] Sundarapandian, V. (2011) "Global chaos synchronization of four-wing chaotic systems by sliding mode control”, International Journal of Control Theory and Computer Modelling, Vol. 1, No. 1, pp. 15-31.

[19] Sundarapandian, V. (2011) "Global chaos synchronization of Pehlivan systems by sliding mode control”, International Journal on Computer Science and Engineering, Vol. 3, No. 5, pp. 21632169.

[20] Sundarapandian, V. (2011) "Sliding mode controller design for the synchronization of ShimizuMorioka chaotic systems", International Journal of Information Sciences and Techniques, Vol. 1, No. 1, pp 20-29. 
[21] Li, G.H. (2005) "Synchronization and anti-synchronization of Colpitts oscillators using active control," Chaos, Solitons \& Fractals, Vol. 26, pp 87-93.

[22] Hu, J. (2005) "Adaptive control for anti-synchronization of Chua's chaotic system", Physics Letters A, Vol. 339, pp. 455-460.

[23] Rössler, O.E. (1979) “An equation for hyperchaos”, Phys. Letters A, Vol. 71, pp 155-157.

[24] Thamilmaran, K., Lakshmanan, M. \& Venkatesan, A. (2004) "A hyperchaos in a modified canonical Chua's circuit”, International J. Bifurcat. Chaos, Vol. 14, pp 221-243.

[25] Vicente, R., Dauden, J., Colet, P. \& Toral, R. (2005) "Analysis and characterization of the hyperchaos generated by a semiconductor laser object", IEEE J. Quantum Electronics, Vol. 41, pp 541-548.

[26] Li, Y., Tang, W.K.S. \& Chen, G. (2005) "Generating hyperchaos via state feedback control," Internat. J. Bifurcat. Chaos, Vol. 15, No. 10, pp 3367-3375.

[27] Wang, J. \& Chen, J. (2008) "A novel hyperchaotic system and its complex dynamics", Internat. J. Bifurcat. Chaos, Vol. 18, No. 11, pp 3309-3324.

[28] Bao, B.C. \& Liu, Z. (2008) "A hyperchaotic attractor coined from the chaotic Lü system”, Chin. Physics Letters, Vol. 25, pp 2396-2399.

[29] Xu, J., Cai, G. \& Zheng, S. (2009) "A novel hyperchaotic system and its control”, J. Uncertain Systems, Vol. 3, pp 137-144.

[30] Hahn, W. (1967) The Stability of Motion, Springer, New York.

\section{Authors}

Dr. V. Sundarapandian obtained his Doctor of Science degree in Electrical and Systems Engineering from Washington University, Saint Louis, USA under the guidance of Late Dr. Christopher I. Byrnes (Dean, School of Engineering and Applied Science) in 1996. He is currently Professor in the Research and Development Centre at Vel Tech Dr. RR \& Dr. SR Technical University, Chennai, Tamil Nadu, India. He has published over 160 refereed international publications. He has published over 90 papers in National Conferences and over 50 papers in International Conferences. He is the Editor-in-Chief of International Journal of Mathematics and Scientific Computing, International Journal of Instrumentation and Control Systems, International Journal of Control Systems and Computer Modelling, etc. His research interests are Linear and Nonlinear Control Systems, Chaos Theory and Control, Soft Computing, Optimal Control, Process Control, Operations Research, Mathematical Modelling, Scientific Computing using MATLAB etc.

Mr. R. Suresh obtained his M.Sc degree in Mathematics from Bharathiar University, Coimbatore, Tamil Nadu, India in 2005, and M.Phil degree in Mathematics from Bharathiar University, Coimbatore, Tamil Nadu, India in 2008. He is currently working as a Lecturer in the Department of Mathematics, Vel Tech Dr. RR \& Dr. SR Technical University, Chennai, India. He is pursuing his Ph.D. degree in Mathematics under the guidance of Research Professor, Dr. V. Sundarapandian in Vel Tech Dr. RR \& Dr. SR Technical University, Chennai, India. He has published over 10 papers in International

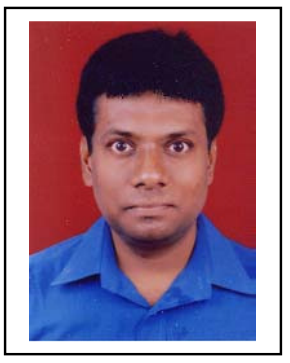
Journals. His research interests are in Chaos and Control Theory. 